\title{
Systematic clinical evidence review of NASHA (Durolane hyaluronic acid) for the treatment of knee osteoarthritis
}

This article was published in the following Dove Press journal: Open Access Rheumatology: Research and Reviews

\author{
Ross Leighton' \\ Jane Fitzpatrick ${ }^{2,3}$ \\ Helen Smith ${ }^{4}$ \\ Daniela Crandall ${ }^{4}$ \\ Carl R Flannery ${ }^{4}$ \\ Thierry Conrozier ${ }^{5}$ \\ 'Department of Surgery, Dalhousie \\ University, Halifax, NS, Canada; \\ ${ }^{2}$ Sports Medicine Professionals \\ Pty Ltd, Richmond, VIC, Australia; \\ ${ }^{3}$ University of Western Australia, \\ Perth, WA, Australia; ${ }^{4}$ Bioventus LLC, \\ Durham, NC, USA; ${ }^{5}$ Department \\ of Rheumatology, Hospital Nord \\ Franche-Comte, Belfort, France
}

\begin{abstract}
Background: Pain and limitations in joint mobility associated with knee osteoarthritis (OA) are clinically challenging to manage, and advanced progression of disease can often lead to total knee arthroplasty. Intra-articular injection of hyaluronic acid (HA), also referred to as viscosupplementation, is a non-surgical treatment approach for OA, the effectiveness of which may depend on the HA composition, and the length of time over which it resides in the joint. One of the available options for such therapies includes NASHA (Durolane HA), a non-animal, biofermentation-derived product, which is manufactured using a process that stabilizes the HA molecules to slow down their rate of degradation and produce a unique formulation with a terminal half-life of $\sim 1$ month. The objectives of the current review were to assess, in patients with OA of the knee, the efficacy and safety of intra-articular treatment with NASHA relative to control (saline) injections, other HA products, and other injectables (corticosteroids, plateletrich plasma, mesenchymal stem cells).
\end{abstract}

Methods: This systematic evidence review examines patient outcomes following NASHA treatment as described in published data from studies conducted in subjects with knee OA. A Preferred Reporting Items for Systematic Reviews and Meta-analyses-compliant literature search strategy yielded 11 eligible clinical studies with a variety of comparator arms. Outcomes assessed at various time points following intra-articular treatment included measures of pain, function, quality of life, and incidence of treatment-related adverse events (AEs).

Results: The available evidence reported for the clinical studies assessed demonstrates sustained and effective relief of knee OA symptoms following a single injection of NASHA. In addition, an excellent biocompatibility profile is observed for NASHA as an intra-articular therapy for $\mathrm{OA}$, as reflected by the low rate of AEs associated with treatment.

Conclusion: Treatment with NASHA is an effective and safe single-injection procedure, which can be beneficial in the clinical management of knee OA.

Keywords: osteoarthritis, viscosupplementation, intra-articular, hyaluronic acid, NASHA, Durolane, residence time, symptomatic relief, clinical outcomes

\section{Background}

Osteoarthritis (OA) is the most common form of arthritis and is a major cause of debilitation for affected individuals. The prevalence of OA increases with age, with a lifetime risk for developing symptomatic knee OA of $\sim 45 \%$, rising to over $60 \%$ in obese individuals. ${ }^{1} \mathrm{OA}$ is also a leading indication for prescription pain drug expenditure, averaging an annual cost per person of approximately US $\$ 3000 .^{2}$ One of the nonsurgical options for the symptomatic management of $\mathrm{OA}$ is intra-articular injection of hyaluronic acid (HA), also referred to as viscosupplementation. HA is a major
Correspondence: Carl R Flannery

Bioventus LLC, 472I Emperor Blvd., Suite 100, Durham, NC 27703, USA

Tel + I 9194746773

Email carl.flannery@bioventusglobal.com 
component of synovial fluid, which helps to facilitate lubrication and shock absorption in joints, and OA is characterized in part by a decrease in HA molecular weight (MW) and concentration in the synovial fluid as the disease progresses, leading to a reduction in viscoelastic properties. ${ }^{3}$

A number of HA products, with distinct compositions, are available for the treatment of knee OA. These products are differentiated by their source of HA and method of production; the $\mathrm{MW}$ of the active ingredient; the amount, concentration, and injection volume per dose; and the number of injections per course of therapy, ranging from a single administration to up to 5 weekly intra-articular injections. Potential clinical shortcomings of HA viscosupplementation may relate to limited persistence of injected HA in the treated joint, as a result of physiological turnover and possibly due to elevated degradation of HA. Several HA formulations incorporate chemical cross-linking to increase molecular size and weight, in an attempt to improve residence time in the joint. A recent overview concluded that intra-articular HA products derived from biological fermentation, and those that have a MW of $\geq 3000$ kilodaltons, may demonstrate superior efficacy in the treatment of knee OA. ${ }^{4}$

NASHA (Durolane HA; Bioventus LLC, Durham, NC, USA) is a biofermentation-derived HA product available in a number of countries for the symptomatic treatment of OA with a single-injection course of therapy. During the NASHA production process, the natural entanglements that exist in HA are augmented by the introduction of a minimal $(\sim 1 \%)$ number of synthetic cross-links. Linear HA molecules are thereby incorporated into a 3-dimensional gel matrix, with optimized rheological properties, that is resistant to degradation. ${ }^{5}$ The resulting formulation remains biocompatible, with an increased density of HA that allows for delivery of a higher dose per unit volume (60 $\mathrm{mg}$ per $3 \mathrm{~mL}$ ).

The residence time of NASHA following injection into knee joints has been determined both in animals and in human subjects. In a rabbit knee model, NASHA was found to have a half-life of 32 days, which is markedly longer than that observed for unmodified HA (half-life of $<1$ day) or the cross-linked HA Hylan G-F 20 (half-life of 1.5 days for the hylan A component representing $90 \%$ of the product, and 8.8 days for hylan B). ${ }^{6}$ In human knee joints, NASHA is the only HA product for which residence time data has been reported, with a measured half-life of 4 weeks. ${ }^{7}$ In the current review, we evaluated published clinical studies of NASHA for the treatment of knee OA, to help further understand how its unique HA chemical structure and properties can contribute to meaningful and sustained relief of knee OA symptoms.

\section{Methods}

A search of literature indexed in MEDLINE/PubMed and published through December 2017 was performed to identify relevant articles for this review on the basis of the Preferred Reporting Items for Systematic Reviews and Meta-Analyses guidelines, ${ }^{8}$ using the following search strategy and terms: (( ((() (clinical trial [Publication Type]) OR clinical trials as topic[MeSH Terms]) OR clinical trial) OR patients)) AND ((Durolane OR NASHA))) AND ((osteoarthritis, knee[MeSH Terms]) OR (knee AND osteoarthritis)). The search was also extended to the Cochrane Library and Google Scholar, and additional publications were identified through literature surveillance by the authors. Publication titles, abstracts, and full text articles were independently screened and assessed for quality of evidence, by 3 reviewers (HS, DC, CRF). Only clinical studies of NASHA that employed a $3 \mathrm{~mL}$ injection volume for the symptomatic treatment of knee OA were included. Studies of NASHA for other joints/indications, as well as other reviews or meta-analyses, were excluded. Levels of clinical evidence were determined for each included study based on published guidance criteria. ${ }^{9}$ The Grades of Recommendation, Assessment, Development, and Evaluation criteria ${ }^{10}$ were used to evaluate risk of bias for applicable studies.

In the clinical studies identified, multiple measures of efficacy were assessed, including the visual analog scale (VAS), Western Ontario and McMaster Universities Osteoarthritis Index (WOMAC), Lequesne Index, pain responder rates, and general patient-reported metrics, such as patient global pain and functional assessments. In studies utilizing the WOMAC pain subscale to measure effectiveness, responders were study subjects who reported at least a $40 \%$ improvement from baseline in the WOMAC pain score, plus a minimum absolute improvement of 5 points for the treated knee.

\section{Results}

A total of 15 full-text publications were identified based on the search criteria and literature surveillance, of which 4 were review articles and were excluded. A systematic clinical evidence review was thus performed for 11 relevant studies (Figure 1). Data reported for changes in pain, joint function, quality of life (QoL), and safety measurements were assessed from 7 Level $\mathrm{I}^{11-17}$ and 4 Level II-III studies of NASHA ${ }^{18,20-22}$ (Table 1). Raw data values for the outcome 

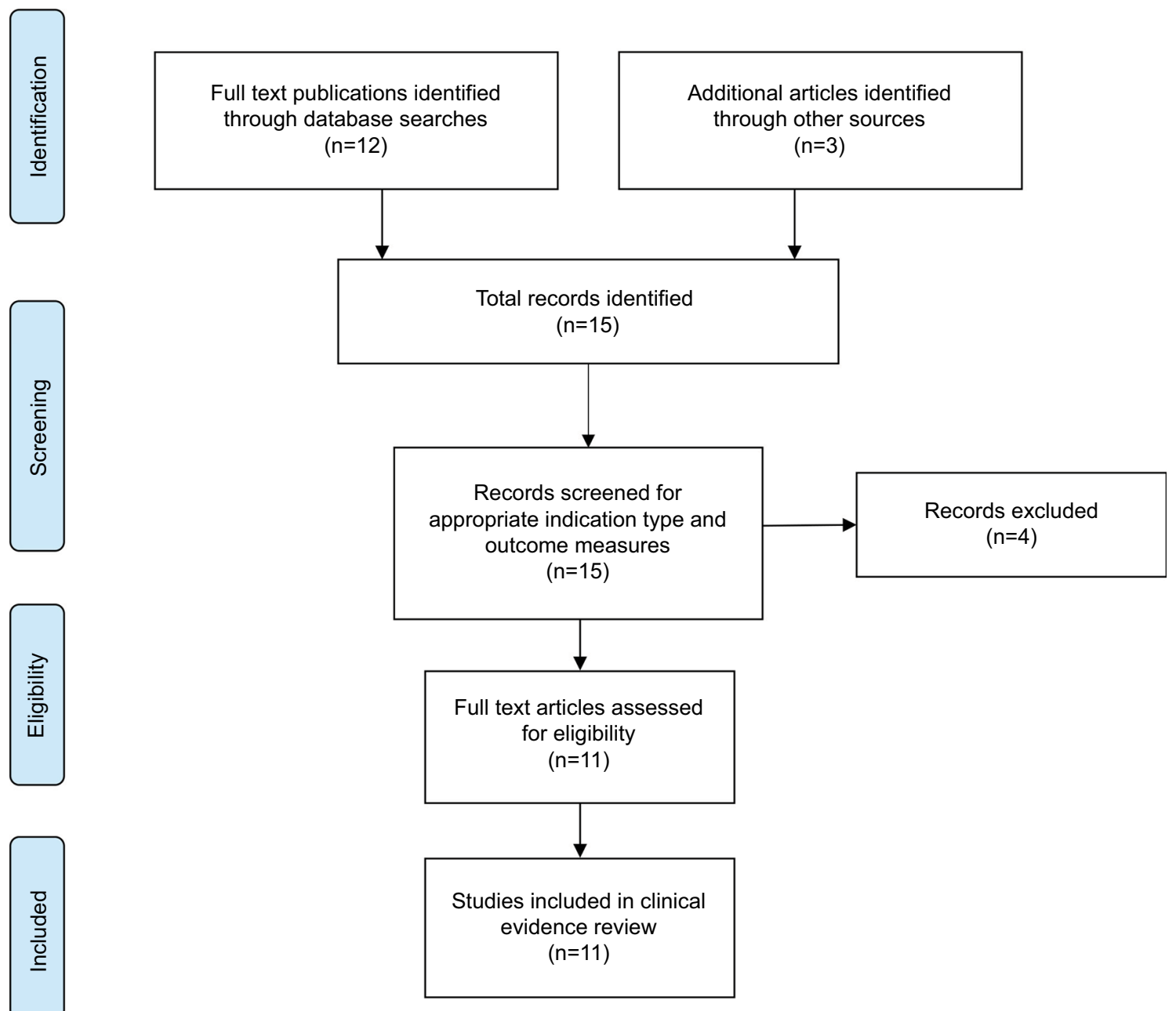

Figure I Flowchart of search and selection process for clinical studies included in review.

measures assessed were derived from the published articles; in the case of 1 study, ${ }^{14}$ unpublished raw data from the clinical study report were included (Table S1). Among the Level I studies, 2 compared NASHA with another HA product; ${ }^{11,12}$ 2 compared NASHA with a corticosteroid; ${ }^{13,14} 2$ compared NASHA with saline treatment; ${ }^{15,16}$ and 1 compared NASHA with allogeneic mesenchymal stem cells (MSCs). ${ }^{17}$ For the Level II-III studies, 1 compared NASHA with a platelet-rich plasma (PRP) preparation; ${ }^{18} 2$ were uncontrolled, observational studies; $;^{20,21}$ and 1 compared NASHA with another HA product. ${ }^{22}$

Of the level I studies, 6 studies ${ }^{12-17}$ demonstrated a low level of risk for the parameters of sequence generation, treatment allocation concealment, blinding of patients and personnel, outcome assessment blinding, incomplete outcome reporting, and selective data reporting. One study ${ }^{11}$ was assigned an uncertain level of risk for treatment allocation concealment, patients and personnel blinding, and outcome assessment blinding, with a low level of risk for the other aforementioned parameters. Four studies reported receiving study funding from a company source. ${ }^{12,14-16}$

\section{Efficacy of NASHA for pain outcomes in knee OA}

Two Level I studies that compared NASHA with another HA product demonstrated significant pain relief with NASHA. ${ }^{11,12}$ One study demonstrated superiority for NASHA relative to single-injection Hylan G-F 20, ${ }^{11}$ while another study found comparable pain relief for NASHA when compared with 5 weekly injections of sodium hyaluronate (Artz). ${ }^{12}$ In the study conducted in 182 subjects comparing NASHA with Hylan G-F 20, improvements in OA-related pain were longer lasting in the NASHA-treated patients, with VAS pain scores significantly more improved at 6 months among the NASHAtreated patients $(P<0.001) .{ }^{11}$ The CHASE trial compared a single injection of NASHA ( $\mathrm{n}=174)$ to 5 weekly injections 
Table I Summary of clinical studies of NASHA for the treatment of knee OA

\begin{tabular}{|c|c|c|c|}
\hline Level I studies & Comparator & Study subjects $(\mathbf{N})$ & Key results for patient symptomatic assessments \\
\hline McGrath et al"I & Hylan G-F 20 & $\begin{array}{l}\text { NASHA or Hylan G-F } 20 \\
\text { randomized (182 knees) }\end{array}$ & $\begin{array}{l}\text { NASHA superior to Hylan G-F } 20 \text { over } 26 \text { weeks for knee pain } \\
\text { and flexion, quality of life, and reduced use of analgesics and } \\
\text { anti-inflammatories. }\end{array}$ \\
\hline Zhang et $\mathrm{al}^{12}$ & Sodium hyaluronate & $\begin{array}{l}\text { NASHA (16I); sodium hyaluronate } \\
(158)\end{array}$ & $\begin{array}{l}\text { NASHA non-inferior to sodium hyaluronate over } 26 \text { weeks for } \\
\text { pain, physical function, stiffness, and global self-assessment. }\end{array}$ \\
\hline Skwara et al ${ }^{13}$ & $\begin{array}{l}\text { Triamcinolone } \\
\text { acetonide, steroid }\end{array}$ & NASHA (24); TA (26) & $\begin{array}{l}\text { NASHA superior to TA steroid over } 12 \text { weeks for knee pain. } \\
\text { NASHA non-inferior to TA steroid over } 12 \text { weeks for knee } \\
\text { function/range of motion. }\end{array}$ \\
\hline Leighton et $\mathrm{al}^{14}$ & $\begin{array}{l}\text { Methylprednisolone } \\
\text { acetate, steroid }\end{array}$ & $\begin{array}{l}\text { Single injection (blinded) phase: } \\
\text { NASHA (22I); MPA (22I) }\end{array}$ & $\begin{array}{l}\text { NASHA non-inferior to MPA steroid over } 26 \text { weeks for } \\
\text { WOMAC pain, responder rates, physical function, and stiffness. } \\
\text { Effect sizes favored NASHA over MPA from } 12 \text { to } 26 \text { weeks. }\end{array}$ \\
\hline Altman et $\mathrm{al}^{15}$ & Saline & $\begin{array}{l}\text { Patients with knee OA only: } \\
\text { NASHA (107); saline (109) }\end{array}$ & $\begin{array}{l}\text { NASHA superior to saline over } 6 \text { weeks for responder rates in } \\
\text { patients with OA confined to the knee. }\end{array}$ \\
\hline Arden et al $^{16}$ & Saline & $\begin{array}{l}\text { Patients without clinical effusion: } \\
\text { NASHA (69); saline (66) }\end{array}$ & $\begin{array}{l}\text { NASHA superior to saline over } 6 \text { weeks for knee pain in } \\
\text { patients without clinical effusion. }\end{array}$ \\
\hline Vega et al ${ }^{17}$ & MSC & NASHA (15); MSCs (15) & $\begin{array}{l}\text { Both NASHA and MSCs significantly improved VAS knee pain } \\
\text { at } 12 \text { months. }\end{array}$ \\
\hline \multicolumn{4}{|l|}{ Level II-III studies } \\
\hline Vaquerizo et al ${ }^{18}$ & PRGF-Endoret & NASHA (48); PRGF (48) & $\begin{array}{l}\text { OMERACT-OARSI responders at } 24 \text { and } 48 \text { weeks: } \\
\text { NASHA }=27 \% \text { and } 21 \% \text {, respectively; PRGF= } 83 \% \text { and } 69 \% \text {, } \\
\text { respectively. }\end{array}$ \\
\hline Akermark et $\mathrm{a}^{20}$ & None (uncontrolled) & $\begin{array}{l}\text { First injection phase: } \\
\text { NASHA (103) } \\
\text { Second injection phase: } \\
\text { NASHA (53) }\end{array}$ & $\begin{array}{l}\text { NASHA treatment significantly improved knee pain at } 3 \text { months } \\
\text { follow-up after first injection, and at I-month follow-up after } \\
\text { second injection. }\end{array}$ \\
\hline Krocker et $\mathrm{al}^{21}$ & None (uncontrolled) & NASHA (50) & $\begin{array}{l}\text { NASHA significantly improved knee pain, quality of life and } \\
\text { activity, and range of motion at } 24 \text { weeks. }\end{array}$ \\
\hline Estades-Rubio et $\mathrm{al}^{22}$ & Sodium hyaluronate & $\begin{array}{l}\text { NASHA (27); sodium hyaluronate } \\
\text { (27) }\end{array}$ & $\begin{array}{l}\text { NASHA superior to sodium hyaluronate at } 26 \text { weeks for knee } \\
\text { pain and stiffness, and functional limitation. }\end{array}$ \\
\hline
\end{tabular}

Abbreviations: NASHA, Durolane hyaluronic acid; MSCs, mesenchymal stem cells; MPA, methylprednisolone acetate; OA, osteoarthritis; OMERACT-OARSI, Outcome Measures in Rheumatology-Osteoarthritis Research Society International; PRGF, plasma rich in growth factors; TA, triamcinolone acetonide; VAS, visual analog scale; WOMAC, Western Ontario and McMaster Universities Osteoarthritis Index.

of sodium hyaluronate $(\mathrm{n}=175) .{ }^{12}$ This non-inferiority study showed that WOMAC pain scores were equally improved from baseline in both treatment groups at 18 and 26 weeks ( $P<0.001$ both groups at both time points), indicating that a single injection of NASHA can be as efficacious in improving OA-related pain as a 5-injection course of unmodified HA. ${ }^{12}$

Two Level I studies compared NASHA with intra-articular corticosteroid treatment of knee OA. ${ }^{13,14}$ In a 12-week study comparing NASHA $(n=24)$ versus a single injection of triamcinolone acetonide (TA; $10 \mathrm{mg} / \mathrm{L} \mathrm{mL}$ dose; $\mathrm{n}=26$ ), VAS pain was shown to be significantly improved from baseline at 12 weeks following NASHA treatment, from $54.9 \mathrm{~mm}$ at baseline to $44 \mathrm{~mm}$ at follow-up $(P=0.0416)$. Patients treated with TA steroid showed no significant improvement in pain at 12 weeks; baseline VAS was 52.9 and $45.8 \mathrm{~mm}$ at followup. ${ }^{13}$ Lequesne scores for both groups improved significantly at 12 weeks relative to baseline. ${ }^{13}$

A 1-year study compared NASHA with a single injection of methylprednisolone acetate (MPA; $40 \mathrm{mg} / \mathrm{L} \mathrm{mL} \mathrm{dose).}{ }^{14} \mathrm{In}$ this study, patients were enrolled if they had OA pain only in the knee and no signs of a clinical effusion in the study knee at baseline. The primary objective was to show non-inferiority of NASHA $(n=221)$ to MPA steroid $(n=221)$ as measured by WOMAC pain responder rates at 12 weeks. Responder rates between the 2 treatment groups were comparable (noninferior) at 12 weeks relative to baseline, and both groups demonstrated similar response rates through week 18 . At 26 weeks, there was a significantly greater Outcome Measures in Rheumatology-Osteoarthritis Research Society International (OMERACT-OARSI) responder rate $(P=0.0237)$, and significantly lower WOMAC pain scores $(P=0.034)$ for NASHA- versus MPA-treated patients. ${ }^{14}$

The 1-year study design of the aforementioned trial ${ }^{14}$ included a 26-week double-blind phase followed by a 26-week open-label, partial cross-over extension (wherein patients could receive NASHA regardless of their initial study arm assignment). At 26 weeks, 342 of 390 subjects chose to receive a second intra-articular treatment with NASHA, of 
whom 163 had received NASHA in the double-blind phase (NASHA+NASHA subgroup) and 179 had received MPA steroid in the double-blind phase (MPA+NASHA subgroup). Improvement in the WOMAC pain response rate was significantly higher for the NASHA+NASHA subgroup at week 39 $(P=0.039)$ and was sustained and similar at week 52 for both subgroups, demonstrating the beneficial effects of a repeat injection of NASHA, as well as the efficacy of NASHA in patients who received a previous treatment with MPA. ${ }^{14}$

Two Level I studies compared NASHA treatment of knee OA to a saline injection control, using the WOMAC pain subscale as an effectiveness endpoint. ${ }^{15,16}$ These 2 studies were retrospectively identified to have incorporated a screening process and study design that led to inadequate study subject homogeneity. Thus, one 26 -week study found no significant difference in responder rates between NASHA $(n=172)$ and saline $(n=174)$ in the overall intention-to-treat (ITT) population. However, when subsets of subjects with multiple sites of OA were excluded, and those with only knee OA were evaluated, NASHA patients $(\mathrm{n}=107)$ demonstrated a significantly greater responder rate at 6 weeks following treatment relative to the saline control group $(\mathrm{n}=109 ; P<0.025) .{ }^{15}$ In patients with unilateral knee OA (e.g., confined to the study knee), an even greater response rate was observed for NASHA $(\mathrm{n}=38)$ compared with saline treatment $(\mathrm{n}=44 ; P<0.0097)$. Another saline-controlled study also found no significant differences between treatment groups ( $\mathrm{n}=108$ for NASHA arm; $\mathrm{n}=110$ for saline arm) at 6 weeks in the main ITT analysis. However, analysis of a subgroup of subjects without clinical effusion in the study knee at baseline showed a significantly higher 6-week pain responder rate with NASHA $(n=69)$ than with saline $(\mathrm{n}=66)$ of $40.6 \%$ versus $19.7 \%$, respectively $(P=0.0084) .{ }^{16}$ These study designs demonstrate the importance of utilizing exclusion criteria, such as polyarticular OA or clinical evidence of joint effusion (e.g., ballotable patella) when screening candidates for a knee OA study of NASHA or other HA products.

Two of the studies reviewed compared NASHA with experimental knee OA treatments, testing either allogeneic $\mathrm{MSCs}^{17}$ or a PRP preparation, plasma rich in growth factors (PRGF-Endoret). ${ }^{18}$ It should be noted, however, that methodological limitations of studies involving patient-derived biological material (e.g., PRP) can preclude the ability to conduct properly blinded trials. For the study comparing NASHA ( $n=15)$ with MSCs $\left(n=15 ; 40 \times 10^{6}\right.$ cells per dose), the effect of NASHA on VAS pain was found to be enduring, with a significant reduction from baseline pain at 12 months $(P<0.05) .{ }^{17}$ Treatment with MSCs also showed a beneficial effect, although baseline pain scores were lower in the MSCtreated arm relative to NASHA-treated patients. ${ }^{17}$ In the study comparing a single injection of NASHA $(n=48)$ with 3 weekly injections of PRGF-Endoret ( $\mathrm{n}=48 ; 8 \mathrm{~mL}$ per dose), patients were randomized but not blinded to treatment. ${ }^{18}$ At 24 and 48 weeks, $27 \%$ and $21 \%$, respectively, of NASHA patients were characterized as OMERACT-OARSI responders, whereas there were $\sim 3$ times more such responders for the PRGF patients. Surprisingly, however, an improvement from baseline in WOMAC pain scores for the NASHA-treated subjects was not detected, contrary to the results of numerous studies showing a substantial and long-term (6-12 months) effect following intra-articular fluid injection. ${ }^{19}$

One uncontrolled study showed significant improvements from baseline values in VAS pain scores $(P<0.0001)$ assessed for various lifestyle scenarios, including at rest and under weight-bearing motion, in 103 NASHA-treated patients evaluated for effectiveness at 3 months following injection. ${ }^{20}$ Fifty-three of the enrolled subjects received a second NASHA injection at 6 months. Again, significant improvements in VAS pain scores $(P<0.0001)$ were observed in the treated knees at 1 month after the second injection. ${ }^{20}$ Results from another uncontrolled study conducted in 50 subjects showed a significant decrease in pain at 24 weeks after NASHA treatment $(P<0.001) .{ }^{21}$ In a Level II-III study comparing NASHA $(n=27)$ with sodium hyaluronate $(\mathrm{Go}-\mathrm{ON}$; $\mathrm{n}=27)$ treatment, there was a significant and superior reduction in VAS knee pain at 26 weeks after NASHA injection $(P<0.01){ }^{22}$

\section{Efficacy of NASHA for physical function and joint stiffness measures in knee OA}

In addition to pain management, improvements in patient mobility and joint function are important objectives in the treatment of knee OA and are included as effectiveness outcomes in many OA clinical trials. Changes in patient physical function and joint stiffness were measured in 8 of the identified studies reviewed here, using a variety of scoring systems $^{11-16,18,21}$ (Table 1).

In the Level I study that compared NASHA with Hylan G-F 20, a significant increase in range of flexion motion was observed for NASHA relative to Hylan G-F 20 for up to 9 months $(P=0.0001) .{ }^{11}$ In the Level I non-inferiority study comparing NASHA with sodium hyaluronate, improvements in WOMAC subscales for physical function and joint stiffness were both significantly improved from baseline at 18 
and 26 weeks $(P<0.001$ for all values $)$, and of comparable magnitude. $^{12}$

The Level I study that compared NASHA with TA steroid used gait analysis and the Knee Society Score (KSS), a composite measure of range of flexion motion and joint alignment and stability, to evaluate functional changes. ${ }^{13}$ The gait analysis revealed no significant difference between treatment groups. The composite KSS score showed a significant improvement at week 12 of 12.2 points in the NASHA group $(P<0.0007)$ and 6.2 points in the TA group $(P=0.0069) .{ }^{13}$

For the Level I study that compared NASHA to MPA steroid, similar improvements in joint function (WOMAC subscales for physical function and joint stiffness) with NASHA and MPA were observed for up to 26 weeks following the first injection (blinded phase) and up to the 52 week timepoint (end of open label extension phase). ${ }^{14}$

One of the uncontrolled studies reported using the Knee and Osteoarthritis Outcome Score (KOOS) as well as range of motion (ROM) to assess patient function following NASHA treatment. ${ }^{21}$ The KOOS measures of general and sports activities were significantly improved from baseline scores at 24 weeks after NASHA treatment $(P<0.001$ and $P<0.05$, respectively). ${ }^{21}$ Active ROM was also significantly improved with NASHA treatment, increasing from $109^{\circ}$ to $115^{\circ}$ at 24 weeks $(P=0.006) .{ }^{21}$ For the Level II-III study comparing NASHA with sodium hyaluronate, knee stiffness and functional limitation scores were significantly superior for NASHA at 26 weeks. ${ }^{22}$

\section{Efficacy of NASHA for QoL assessments in knee OA}

Patient QoL assessments are an increasingly important measure of the effectiveness of patient-centered care accompanying a medical treatment. ${ }^{23}$ QoL is, therefore, an important outcome to consider in clinical evaluations and was measured in 5 of the studies analyzed in this review. ${ }^{11-13,17,21}$

The Level I study that compared outcomes for a single injection of NASHA or Hylan G-F 20 used the SF-36 version 2 Mental Component Score (MCS) to measure changes in mental status. Both treatments demonstrated a significant improvement in MCS from baseline at 3 months $(P<0.001)$, which persisted for up to 12 months for NASHA-treated subjects; at 12 months the MCS for NASHA treatment was significantly higher than for Hylan G-F 20 treatment $(P=0.005) .{ }^{11}$

In the Level I study comparing NASHA to 5 weekly injections of sodium hyaluronate, self-reported global assessments of improvement from baseline were significant in both treatment groups at weeks 18 and $26(P<0.001){ }^{12}$

The study comparing NASHA versus TA steroid used the SF-36 questionnaire to evaluate self-reported outcomes. Subjects treated with NASHA reported improvements from baseline in all eight domains, particularly the Physical Role and Emotional Role. There were no significant differences in SF-36 domain scores between the NASHA and TA treated groups. ${ }^{13}$

In the study comparing knee OA treatment with NASHA versus allogeneic MSCs, there were no significant differences between treatments detected by SF-36 and SF-12 (Physical Component and MCSs) questionnaires. ${ }^{17}$

In one of the uncontrolled studies of NASHA, QoL was measured by the EQ-5D questionnaire, with domains for mobility, self-sufficiency, daily activities, pain, and mood. A significant improvement from baseline EQ-5D scores was seen with NASHA at 2 and 24 weeks $(P<0.001){ }^{21}$

Another endpoint when assessing QoL is the time until there is need for surgical intervention for knee OA. One retrospective study of outcomes in a knee OA clinic found that the time to total knee arthroplasty (TKA) was 1093 days (95\% CI, 980-1206) among patients who received viscosupplementation with NASHA versus 694 days (95\% CI, 548-839) among the patients who did not receive viscosupplementation. ${ }^{24}$ In an analysis of a subgroup of patients with Grade 3 severity of knee OA on the Kellgren-Lawrence (KL) scale, the time to TKA was 1278 days (95\% CI, 474-2081) with NASHA versus 596 days (95\% CI, 14-1179) without viscosupplementation. ${ }^{24}$

\section{Safety of NASHA treatment for knee OA}

NASHA is a biocompatible formulation of non-animal $\mathrm{HA}$, and, therefore, has a decreased risk for contamination or adverse reactions. As with other HA formulations that are biofermentation-derived, it is expected that NASHA would have an excellent safety profile, with fewer reports of adverse events (AEs) at the injection site than with some avian-derived HA product(s). ${ }^{5}$ In support of this, preclinical studies have observed more aggressive tissue reactions to avian-derived cross-linked HA (Hylan G-F 20) than for NASHA. ${ }^{25}$

Seven of the clinical studies included in this review reported AEs. Treatment-related AEs (TRAEs) were all local and transient, and most were mild (Table 2). ${ }^{12,14-16,18,20,21}$ In the Level I study comparing NASHA with sodium hyaluronate, ${ }^{12} 23$ of the 175 subjects treated with NASHA (13.1\%) 
Table 2 Incidence of TRAEs reported in clinical studies of NASHA for the treatment of knee OA

\begin{tabular}{|c|c|c|}
\hline & TRAE incidence & Category of TRAE \\
\hline \multicolumn{3}{|l|}{ Level I studies } \\
\hline Zhang et al ${ }^{12}$ & $13.1 \%$ & Arthralgia, joint swelling \\
\hline \multirow[t]{5}{*}{ Leighton et a $\left.\right|^{14}$} & $17.2 \%, 18.4 \%^{a}$ & Arthralgia \\
\hline & $1.4 \%, 0 \%{ }^{\mathrm{a}}$ & Injection site pain \\
\hline & $1.8 \%, 0.6 \%^{\mathrm{a}}$ & Joint stiffness \\
\hline & $2.3 \%, 1.2 \%^{\mathrm{a}}$ & Joint swelling \\
\hline & $0 \%, 1.8 \%^{a}$ & Musculoskeletal discomfort \\
\hline Altman et $\mathrm{al}^{15}$ & $12.7 \%$ & Arthralgia \\
\hline Arden et al ${ }^{16}$ & $15.7 \%$ & Knee pain, swelling \\
\hline \multicolumn{3}{|c|}{ Level II-III studies } \\
\hline \multirow[t]{2}{*}{ Vaquerizo et al ${ }^{18}$} & $12.5 \%$ & Post-injection pain \\
\hline & $4.1 \%$ & Pseudoseptic reaction \\
\hline Akermark et $\mathrm{a}^{20}$ & $5 \%, 4 \%^{\mathrm{b}}$ & Knee pain, swelling \\
\hline Krocker et $\mathrm{al}^{21}$ & $18.0 \%$ & Joint swelling \\
\hline
\end{tabular}

Notes: a ${ }^{2}$ alues for the randomized phase, and the open-label phase. ${ }^{b}$ Values for the first injection, and the second injection 6 months later.

Abbreviations: NASHA, Durolane hyaluronic acid; OA, osteoarthritis; TRAE, treatment-related adverse event.

reported treatment-related arthralgia, injection site pain, or joint swelling. The safety of NASHA was also evaluated in a 1-year Level I study: ${ }^{14}$ for the first 26 weeks, treatment was double-blinded and randomized to NASHA or MPA steroid, while treatment for the following 26 weeks was open-label with NASHA. Most TRAEs were reported within 3 days of treatment and resolved in 2-3 weeks. The incidence of TRAEs among the 221 subjects treated with NASHA during the double-blind phase was: arthralgia, $17.2 \%$; injection site pain, 1.4\%; joint stiffness, $1.8 \%$; and joint swelling, $2.3 \%$. During the open-label phase, the type and incidence of TRAEs among the 342 subjects treated with NASHA was: arthralgia, $18.4 \%$; joint stiffness, $0.6 \%$; joint swelling, $1.2 \%$; and musculoskeletal discomfort, $1.8 \% .{ }^{14}$ Importantly, a repeat injection of NASHA did not lead to the occurrence of any allergic reactions or observations of treatment-related serious AEs.

In one of the saline-controlled studies, 22 of 173 NASHAtreated subjects reported a TRAE (12.7\%), most commonly arthralgia, all within 2 days of treatment. ${ }^{15}$ In the other salinecontrolled study, ${ }^{16}$ there were 17 TRAEs reported among the 108 NASHA-treated patients (15.7\%). Most were mild and all involved either post-injection pain or transient knee swelling. ${ }^{16}$ Among the Level II-III studies, 1 study reported that 8 of 48 subjects experienced mild-to-moderate TRAEs $(16.6 \%)$, mostly attributed to post-injection pain. ${ }^{18}$ One of the uncontrolled studies of NASHA was designed principally to measure tolerability of the HA treatment. ${ }^{20} \mathrm{After}$ the first injection, 7 unanticipated TRAEs of knee pain or swelling were reported among the 128 knees evaluated for tolerability
$(5 \%) .{ }^{20}$ Following the second injection at 6 months, there were 3 similar TRAEs among the 72 knees evaluated for tolerability (4\%). ${ }^{20}$ In the other uncontrolled study of NASHA, there were 9 cases (18\%) of knee joint swelling without hyperthermia. ${ }^{21}$

\section{Physician perspectives: treatment of knee OA with NASHA in clinical practice}

In clinical practice, if a symptomatic patient with KL Grade 2 or 3 OA presents with knee pain, first-line therapy typically comprises treatment with a non-steroidal anti-inflammatory drug, and potentially an intra-articular injection of a corticosteroid to reduce effusion. A review of the patient 8 to 12 weeks later is conducted to assess treatment success, or to determine whether short-term pain alleviation (e.g., for 6-8 weeks) has waned and pain has returned. The latter patient characteristics indicate an appropriate candidate for treatment with a high MW, non-avian-based HA injection, such as NASHA. Based on the review of the clinical data reported herein for NASHA, successful pain relief would be expected to last for at least 6-9 months following such treatment. Repeat injections would be expected to yield a similar result, at a minimum, with longer effectiveness anticipated in the majority of patients who were responsive to initial treatment. A case study from one of the authors (RL) demonstrates this concept in practice for a 58-year-old male with painful bilateral knee OA (Figure 2) who received initial treatment with MPA steroid. Despite initial pain relief, symptoms returned after 6-8 weeks. Subsequent treatment with NASHA was effective for 9-12 months, and the patient has received yearly injections of NASHA for the past 5 years with beneficial results.

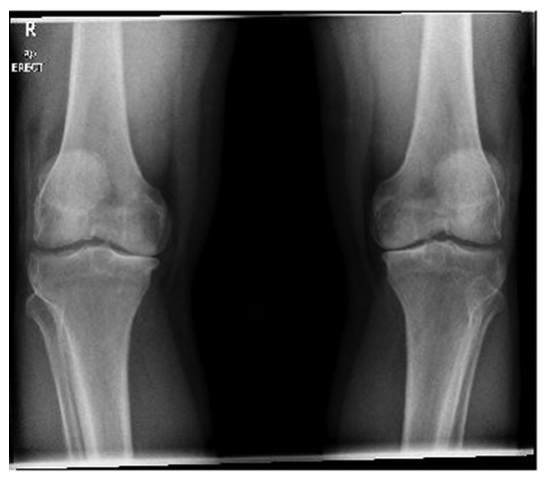

Figure 2 Standing anteroposterior radiograph of a 58-year-old male patient with significant bilateral narrowing of the medial aspect of both knees (femorotibial joints), illustrative of loss of the articular cartilage with advancing osteoarthritis. 


\section{Discussion}

The major therapeutic goals for the treatment of knee OA are to decrease joint pain and stiffness, improve patient function and QoL, and delay or avoid the need for TKA. The association of HA injections with prolonging the time to TKA has been illustrated in several studies utilizing independent patient health care databases ${ }^{26-28}$ while another report demonstrated that treatment specifically with NASHA prolonged the time to TKA in subjects with KL Grade 3 knee OA by 3.5 years relative to patients who did not receive viscosupplementation. ${ }^{24}$

NASHA has been shown to be effective in relieving joint pain in preclinical animal models ${ }^{29,30}$ and has been demonstrated to have a half-life of 32 days in rabbit knee joints. ${ }^{6}$ In the only human study reporting on residence time for HA injected into the knee, a half-life for NASHA of 4 weeks was observed. ${ }^{7}$ In the current review, we evaluated published clinical data to examine whether the unique chemical characteristics and features of NASHA, such as prolonged joint residence time, may translate and contribute to enduring symptomatic relief for the treatment of knee OA patients.

Based on the clinical outcomes reported in the 11 studies included in this review, significant reductions from baseline pain, as well as improvements in physical function and joint stiffness from baseline levels, were observed at 26 weeks after a single injection of NASHA in 3 studies, ${ }^{11,12,14}$ and after 24 weeks in a fourth study. ${ }^{21}$ Self-reported enhancements in QoL following NASHA treatment were also found to be significant over an extended time frame..$^{11,12,21}$ Importantly, NASHA was found to have a favorable safety and tolerability profile, with a low incidence of reported, transient TRAEs.

A potential limitation of this review relates to the relatively low number of clinical studies comparing NASHA with other HA products for the treatment of knee OA. However, there are overall few clinical studies for any of the HA formulations indicated for a single-injection course of therapy for knee OA. On the other hand, a major strength of our analysis was the inclusion of 7 level-I studies, with a variety of trial comparator arms, which assessed multiple efficacy measures.

\section{Conclusion}

Given the prevalence of knee OA, which is projected to increase substantially in aging populations, there is a clear need for effective conservative therapies to improve patients' QoL and delay the time to TKA. The results of this clinical evidence review demonstrate that NASHA is most effective in reducing pain and improving function in patients with noninflammatory, unilateral, mild-to-moderate knee OA. Since
NASHA is administered as a single injection, patient visits and associated costs, as well as potential risks associated with multiple injection courses, are reduced. The effects of NASHA treatment are long-lasting and likely to extend the length of time for non-operative treatment and increase the time the patient can live comfortably without undergoing joint replacement surgery.

\section{Acknowledgments}

Medical writing contributions were provided by Carol Gorman, PharmConsultant, Cary, NC, USA. Financial support for this research was provided by Bioventus LLC.

\section{Author contributions}

All authors contributed toward data analysis, drafting and revising the paper and agree to be accountable for all aspects of the work.

\section{Disclosure}

HS, DC, and CRF are employees and RL, JF, and TC are consultants of Bioventus LLC. The authors report no other conflicts of interest in this work.

\section{References}

1. Murphy L, Schwartz TA, Helmick CG, et al. Lifetime risk of symptomatic knee osteoarthritis. Arthritis Rheum. 2008;59(9):1207-1213.

2. White AG, Birnbaum HG, Janagap C, Buteau S, Schein J. Direct and indirect costs of pain therapy for osteoarthritis in an insured population in the United States. J Occup Environ Med. 2008;50(9):998-1005.

3. Wen DY. Intra-articular hyaluronic acid injections for knee osteoarthritis. Am Fam Physician. 2000;62(3):565-570.

4. Altman RD, Bedi A, Karlsson J, Sancheti P, Schemitsch E. Product differences in intra-articular hyaluronic acids for osteoarthritis of the knee. Am J Sports Med. 2016;44(8):2158-2165.

5. Ågerup B, Berg P, Åkermark C. Non-animal stabilized hyaluronic acid: a new formulation for the treatment of osteoarthritis. Bio Drugs. 2005;19(1):23-30.

6. Edsman K, Hjelm R, Lärkner H, et al. Intra-articular duration of Durolane ${ }^{\mathrm{TM}}$ after single injection into the rabbit knee. Cartilage. 2011;2(4): 384-388.

7. Lindqvist U, Tolmachev V, Kairemo K, Aström G, Jonsson E, Lundqvist H. Elimination of stabilised hyaluronan from the knee joint in healthy men. Clin Pharmacokinet. 2002;41(8):603-613.

8. Moher D, Liberati A, Tetzlaff J, Altman DG; PRISMA Group. Preferred reporting items for systematic reviews and meta-analyses: the PRISMA statement. BMJ. 2009;339:b2535.

9. Slobogean G, Bhandari M. Introducing levels of evidence to the Journal of Orthopaedic Trauma: implementation and future directions. J Orthop Trauma. 2012;26(3):127-128.

10. Atkins D, Best D, Briss PA, et al. Grading quality of evidence and strength of recommendations. BMJ. 2004;328:1490-1497.

11. McGrath AF, McGrath AM, Jessop ZM, et al. A comparison of intraarticular hyaluronic acid competitors in the treatment of mild to moderate knee osteoarthritis. J Arthritis. 2013;2:108.

12. Zhang $\mathrm{H}$, Zhang $\mathrm{K}$, Zhang $\mathrm{X}$, et al. Comparison of two hyaluronic acid formulations for safety and efficacy (CHASE) study in knee osteoarthritis: a multicenter, randomized, double-blind, 26-week non-inferiority trial comparing Durolane to Artz. Arthritis Res Ther. 2015;17:51. 
13. Skwara A, Ponelis R, Tibesku CO, Rosenbaum D, Fuchs-Winkelmann S. Gait patterns after intraarticular treatment of patients with osteoarthritis of the knee-hyaluronan versus triamcinolone: a prospective, randomized, doubleblind, monocentric study. Eur J Med Res. 2009;14(4):157-164.

14. Leighton R, Åkermark C, Therrien R, et al; DUROLANE Study Group. NASHA hyaluronic acid vs methylprednisolone for knee osteoarthritis: a prospective, multi-center, randomized, non-inferiority trial. Osteoarthritis Cartilage. 2014;22(1):17-25.

15. Altman RD, Åkermark C, Beaulieu AD, Schnitzer T; Durolane International Study Group. Efficacy and safety of a single intra-articular injection of non-animal stabilized hyaluronic acid (NASHA) in patients with osteoarthritis of the knee. Osteoarthritis Cartilage. 2004;12(8):642-649.

16. Arden NK, Åkermark C, Andersson M, Todman MG, Altman RD. A randomized saline-controlled trial of NASHA hyaluronic acid for knee osteoarthritis. Curr Med Res Opin. 2014;30(2):279-286.

17. Vega A, Martin-Ferrero MA, Del Canto F, et al. Treatment of knee osteoarthritis with allogeneic bone marrow mesenchymal stem cells: a randomized controlled trial. Transplantation. 2015;99(8):1681-1690.

18. Vaquerizo V, Plasencia MÁ, Arribas I, et al. Comparison of intraarticular injections of plasma rich in growth factors (PRGF-Endoret) versus Durolane hyaluronic acid in the treatment of patients with symptomatic osteoarthritis: a randomized controlled trial. Arthroscopy. 2013;29(10):1635-1643.

19. Altman RD, Devji T, Bhandari M, Fierlinger A, Niazi F, Christensen R. Clinical benefit of intra-articular saline as a comparator in clinical trials of knee osteoarthritis treatments: a systematic review and meta-analysis of randomized trials. Semin Arthritis Rheum. 2016;46(2):151-159.

20. Åkermark C, Berg P, Björkman A, Malm P. Non-animal stabilized hyaluronic acid in the treatment of osteoarthritis of the knee: a tolerability study. Clin Drug Investig. 2002;22(3):157-166.

21. Krocker D, Matziolis G, Tuischer J, et al. Die einmalige intraartikuläre Injektion eines synthetischen Hyaluronsäurepräparates reduziert arthroseassoziierte Kniegelenkschmerzen. [Reduction of arthrosis associated knee pain through a single intra-articular injection of synthetic hyaluronic acid]. Z Rheumatol. 2006;65(4):327-331. German.
22. Estades-Rubio FJ, Reyes-Martin A, Morales-Marcos V, et al. Knee viscosupplementation: cost-effectiveness analysis between stabilized hyaluronic acid in a single injection versus five injections of standard hyaluronic acid. Int J Mol Sci. 2017;18(3):E658.

23. Bauman AE, Fardy HJ, Harris PG. Getting it right: why bother with patient-centred care? Med J Aust. 2003;179(5):253-256.

24. Romero Jurado M, Enrique Fidalgo A, Rodríguez Villar V, Mar Medina J, Soler López B. Factors related with the time to surgery in waiting-list patients for knee prostheses. Reumatol Clin. 2013;9(3): 148-155.

25. Wooley PH, Song Z, Harrison A. Hyaluronic acid viscosupplements from avian and non-mammalian sources exhibit biocompatibility profiles with unique, source-specific, antigenic profiles. J Biomed Mater Res B Appl Biomater. 2012;100(3):808-816.

26. Altman R, Lim S, Steen RG, Dasa V. Hyaluronic acid injections are associated with delay of total knee replacement surgery in patients with knee osteoarthritis: evidence from a large US health claims database. PLoS One. 2015;10(12):e0145776.

27. Altman R, Fredericson M, Bhattacharyya SK, et al. Association between hyaluronic acid injections and time-to-total knee replacement surgery. J Knee Surg. 2016;29(7):564-570.

28. Ong KL, Anderson AF, Niazi F, Fierlinger AL, Kurtz SM, Altman RD. Hyaluronic acid injections in medicare knee osteoarthritis patients are associated with longer time to knee arthroplasty. J Arthroplasty. 2016;31(8):1667-1673.

29. Plaas A, Li J, Riesco J, Das R, Sandy JD, Harrison A. Intraarticular injection of hyaluronan prevents cartilage erosion, periarticular fibrosis and mechanical allodynia and normalizes stance time in murine osteoarthritis. Arthritis Res Ther. 2011;13(2):R46.

30. Boettger MK, Kummel D, Harrison A, Schaible HG. Evaluation of longterm antinociceptive properties of stabilized hyaluronic acid preparation (NASHA) in an animal model of repetitive joint pain. Arthritis Res Ther. 2011;13(4):R110. 


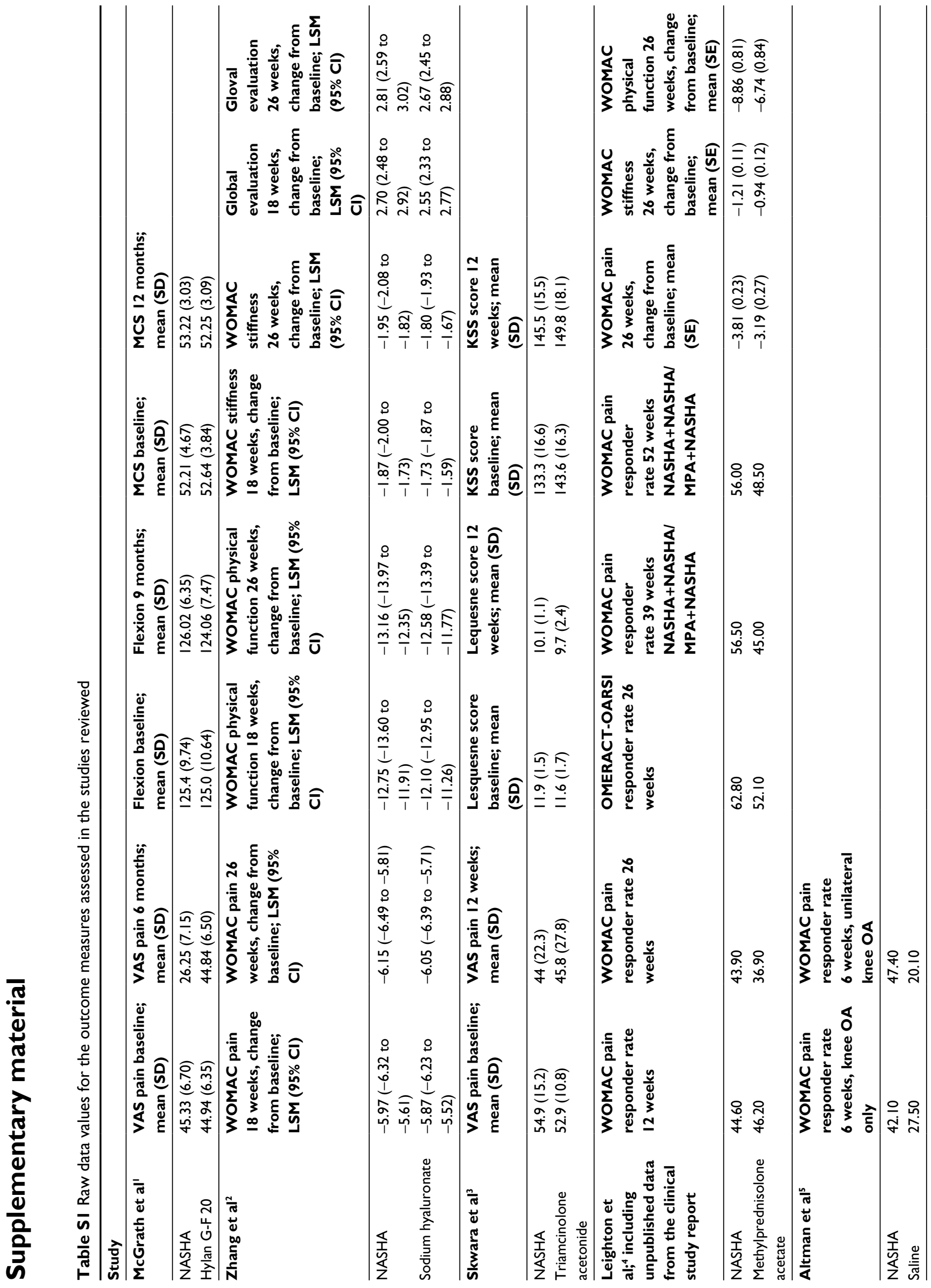




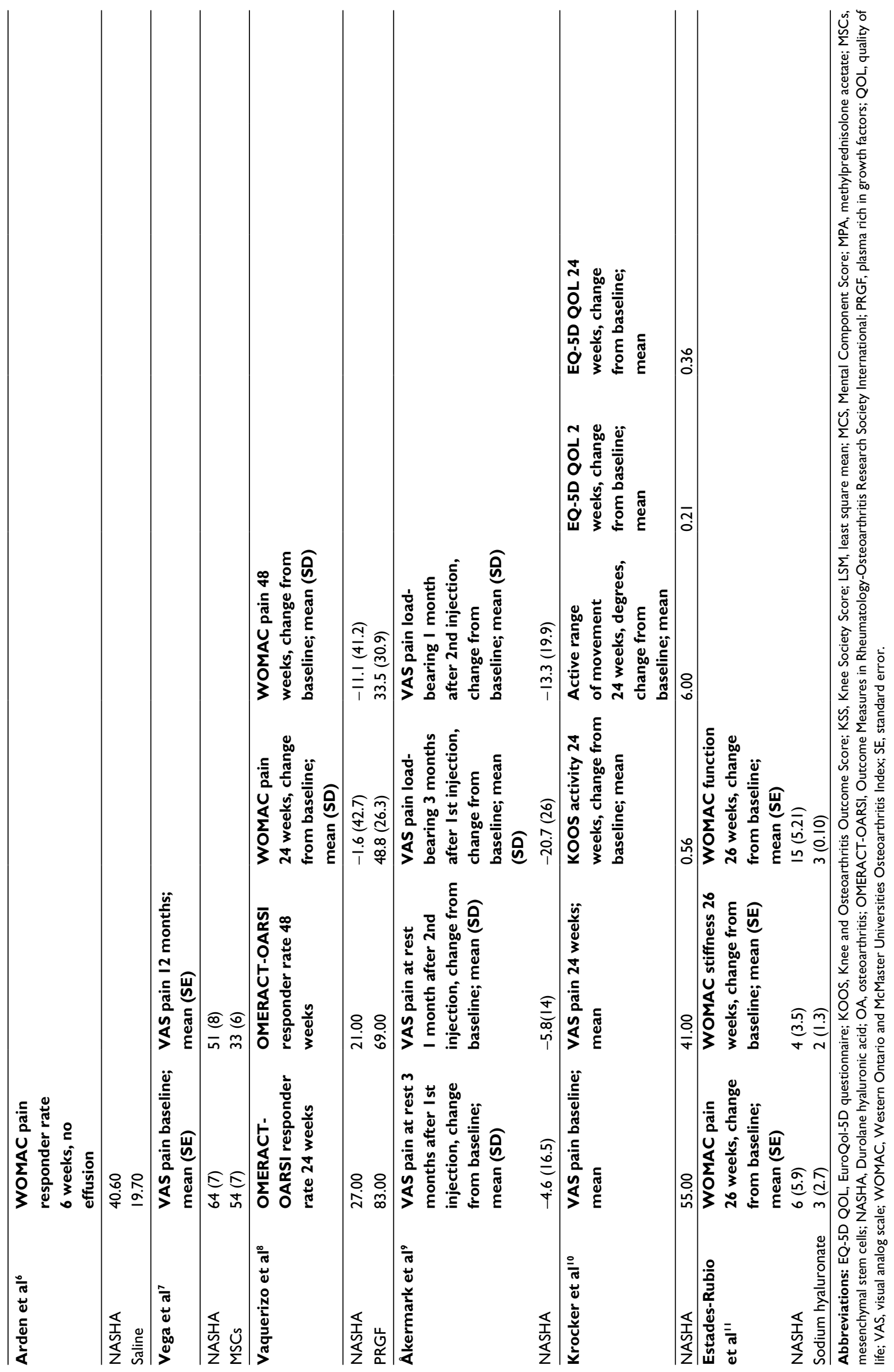




\section{References}

1. McGrath AF, McGrath AM, Jessop ZM, et al. A comparison of intraarticular hyaluronic acid competitors in the treatment of mild to moderate knee osteoarthritis. J Arthritis. 2013;2:108.

2. Zhang H, Zhang K, Zhang X, et al. Comparison of two hyaluronic acid formulations for safety and efficacy (CHASE) study in knee osteoarthritis: a multicenter, randomized, double-blind, 26-week non-inferiority trial comparing Durolane to Artz. Arthritis Res Ther. 2015;17:51.

3. Skwara A, Ponelis R, Tibesku CO, Rosenbaum D, Fuchs-Winkelmann $\mathrm{S}$. Gait patterns after intraarticular treatment of patients with osteoarthritis of the knee-hyaluronan versus triamcinolone: a prospective, randomized, doubleblind, monocentric study. Eur J Med Res. 2009;14(4):157-164.

4. Leighton R, Åkermark C, Therrien R, et al; DUROLANE Study Group. NASHA hyaluronic acid vs methylprednisolone for knee osteoarthritis: a prospective, multi-center, randomized, non-inferiority trial. Osteoarthritis Cartilage. 2014;22(1):17-25.

5. Altman RD, Åkermark C, Beaulieu AD, Schnitzer T; Durolane International Study Group. Efficacy and safety of a single intraarticular injection of non-animal stabilized hyaluronic acid (NASHA) in patients with osteoarthritis of the knee. Osteoarthritis Cartilage. 2004;12(8):642-649.
6. Arden NK, Åkermark C, Andersson M, Todman MG, Altman RD. A randomized saline-controlled trial of NASHA hyaluronic acid for knee osteoarthritis. Curr Med Res Opin. 2014;30(2):279-286.

7. Vega A, Martin-Ferrero MA, Del Canto F, et al. Treatment of knee osteoarthritis with allogeneic bone marrow mesenchymal stem cells: a randomized controlled trial. Transplantation. 2015;99(8):1681-1690.

8. Vaquerizo V, Plasencia MÁ, Arribas I, et al. Comparison of intraarticular injections of plasma rich in growth factors (PRGF-Endoret) versus Durolane hyaluronic acid in the treatment of patients with symptomatic osteoarthritis: a randomized controlled trial. Arthroscopy. 2013;29(10):1635-1643.

9. Åkermark C, Berg P, Björkman A, Malm P. Non-animal stabilized hyaluronic acid in the treatment of osteoarthritis of the knee: a tolerability study. Clin Drug Investig. 2002;22(3):157-166.

10. Krocker D, Matziolis G, Tuischer J, et al. Die einmalige intraartikuläre Injektion eines synthetischen Hyaluronsäurepräparates reduziert arthroseassoziierte Kniegelenkschmerzen. [Reduction of arthrosis associated knee pain through a single intra-articular injection of synthetic hyaluronic acid]. Z Rheumatol. 2006;65(4):327-331. German.

11. Estades-Rubio FJ, Reyes-Martin A, Morales-Marcos V, et al. Knee viscosupplementation: cost-effectiveness analysis between stabilized hyaluronic acid in a single injection versus five injections of standard hyaluronic acid. Int J Mol Sci. 2017;18(3):E658.
Open Access Rheumatology: Research and Reviews

\section{Publish your work in this journal}

Open Access Rheumatology: Research and Reviews is an international, peerreviewed, open access journal publishing original research, reports, editorials, reviews and commentaries on all aspects of clinical and experimental rheumatology in the clinic and laboratory including the following topics: Pathology, pathophysiology of rheumatological diseases; Investigation, treatment and

\section{Dovepress}

management of rheumatological diseases; Clinical trials and novel pharmacologi$\mathrm{cal}$ approaches for the treatment of rheumatological disorders. The manuscript management system is completely online and includes a very quick and fai peer-review system, which is all easy to use. Visit http://www.dovepress.com testimonials.php to read real quotes from published authors. 